\title{
Analyzing energy-efficient configurations in hexapod robots for demining applications
}

\author{
D. Sanz-Merodio, E. Garcia and P. Gonzalez-de-Santos
}

\begin{abstract}
Purpose - Reducing energy consumption in walking robots is an issue of great importance in field applications such as humanitarian demining so as to increase mission time for a given power supply. The purpose of this paper is to address the problem of improving energy efficiency in statically stable walking machines by comparing two leg, insect and mammal, configurations on the hexapod robotic platform SILO6.

Design/methodology/approach - Dynamic simulation of this hexapod is used to develop a set of rules that optimize energy expenditure in both configurations. Later, through a theoretical analysis of energy consumption and experimental measurements in the real platform SILO6, a configuration is chosen.

Findings - It is widely accepted that the mammal configuration in statically stable walking machines is better for supporting high loads, while the insect configuration is considered to be better for improving mobility. However, taking into account the leg dynamics and not only the body weight, different results are obtained. In a mammal configuration, supporting body weight accounts for 5 per cent of power consumption while leg dynamics accounts for 31 per cent.

Originality/value - As this paper demonstrates, the energy expended when the robot walks along a straight and horizontal line is the same for both insect and mammal configurations, while power consumption during crab walking in an insect configuration exceeds power consumption in the mammal configuration.
\end{abstract}

Keywords Robots, Energy consumption, Design, Energy efficiency, Hexapod robots, Insect and mammal configurations, Humanitarian demining

Paper type Research paper

\section{Introduction}

Detection and deactivation of antipersonnel land mines is a very important worldwide concern. It is estimated that, more than 110 million mines are deployed all around the world and cause about 26,000 casualties per year (Gray and Murray, 2003). The large number of active mines, the current low rate of demining and the danger to human operators are consistent reasons to seek an automatic or semi-automatic device capable of detecting mines. Robots based on wheels or tracks can carry sensors over infested fields and accomplish demining tasks effectively. Wheeled robots are the simplest and cheapest and tracked robots are very good for moving over almost all kinds of terrain. Many manned wheeled vehicles or robotic systems have already been tested for humanitarian demining activities (Rizo et al., 2003); however, legged vehicles exhibit interesting potential advantages for demining.

A legged robot only needs a discrete number of groundcontact points, therefore if an antipersonnel mine is detected, it has a greater likelihood of going farther than wheeled or tracked robots. This feature allows all potential alarms in a field to be found before the removal task begins. Legged robots have better omnidirectional mobility than wheeled or tracked robots. Additionally, legged robots have mobility over obstacles, ditches and even stairs, so they can operate in both structured and unstructured environments.

The initiative of applying legged mechanisms for humanitarian demining has been under development for about a decade. At the beginning some general purpose robots were tested for this application but nowadays specific prototypes developing special features are being built and tested. The TITAN VIII walking robot, a four-legged robot developed as a general-purpose walking robot at the Tokyo Institute of Technology, Japan, was one of the first robots adapted for demining tasks (Hirose and Kato, 1998). TITAN VIII used mine detectors attached to its feet. This is a good solution for crossing a minefield, but it is inefficient in detecting all of the mines in a field.

AMRU-2, an electro-pneumatic hexapod developed by the Royal Military Academy and the Free University of Brussels, Belgium (Baudoin et al., 1999), and RIMHO-2, a four-legged robot developed at the Spanish National Research Council (CSIC) (Jimenez and Gonzalez de Santos, 1996), Spain, are two more examples of walking robotic platforms in experimental use for humanitarian demining tasks. AMRU-2 and RIMHO-2 used a front mine-detecting set with very low mobility. An independent

This work has been funded by the Spanish Ministry for Innovation and Science through grant DPI2010-18702. 
scanning manipulator was necessary to improve mine detection over irregular terrain.

COMET-I is perhaps the first legged robot purposefully developed for humanitarian demining missions. It is a sixlegged robot developed at Chiba University, Japan, which incorporates different sensors and location systems (Nonami et al., 2000). Nevertheless, this robot weighs about $120 \mathrm{~kg}$, very heavy for turning out to be real missions, taking into account the directive of the International Test and Evaluation Program for Humanitarian Demining which states that a demining platform must be "small enough to be portable (by manned ground transportation to access the minefield or to be removed from the minefield in case of failure), easy to transport and deploy" (Baudoin, 2005). The Chiba University group has developed a fourth version of this robot, COMET-IV, which weighs about 1 ton (Oku et al., 2008). ARIEL is another hexapod robot, developed by the iRobot Company, for mine-recovery operations (Voth, 2002). In recent years, the Tokyo Institute of Technology has developed TITAN IX, a quadruped robot that uses its legs and feet as manipulators for land-mine removal (Hirose et al., 2005). Continuing the trend in developing walking robots for humanitarian demining activities (Gonzalez de Santos et al., 2006, 2003), the Centre for Automation and Robotics CARCSIC created the DYLEMA project, whose main aim is to develop a locomotion system to integrate relevant technologies in the fields of legged robots as well as sensors to address needs in humanitarian demining activities. The robot developed as a mobile platform for this project was the six-legged robot SILO6 (Figure 1).

All of the platforms previously mentioned have focused their research on the mechanical design and gait generation for the envisaged application. However, there is no related work on the problem of power efficiency. The application of humanitarian demining requires working for several days. Thus, the problem of increase energy efficiency needs to be addressed. This is an old issue in walking mechanisms. In the decade of the eighties the first steps began with the works of Waldron et al. (1984). Afterwards, other analysis have been performed, mostly theoretical (Silva and Machado, 2008; Gonzalez de Santos et al., 2009). This paper, therefore, presents a comparative analysis of the power consumption of two classic leg configurations on the robotic platform SILO6. It is widely accepted that the mammal configuration consumes less energy than the insect one (Todd, 1985). Through a theoretical analysis based on dynamic simulation and real experiments this paper analyzes the role of leg

Figure 1 DYLEMA configuration

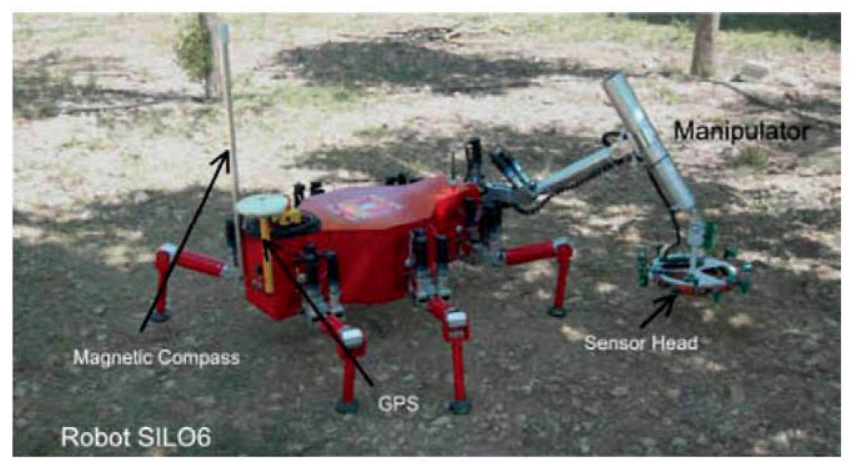

dynamics in the energy consumption of hexapod robots in the two configurations.

The paper is organized as follows: first, Section 2 introduces a complete description of the project. Section 3 analyzes both leg configurations. Section 4 presents an optimization of the chosen critical parameters. Sections 5 and 6 show the results of theoretical and experimental analysis. Finally, Section 7 summarizes the main results and presents some conclusions.

\section{System description}

\subsection{The SILO6 walking robot}

The walking robot, SILO6, is a six-legged-autonomous robot carrying a scanning manipulator, which handles a sensor head composed of a metal detector and a set of infra-red groundtracking sensors (Figure 1). Table I shows the main features of this walking robot.

The main task of the body of the SILO6 walking robot is accommodating legs and subsystems. Therefore, it must be big enough to contain the required subsystems: on-board computer, electronics, drivers, DGPS, batteries, etc. The robot motion is performed by means of an alternating-tripod gait, which means that two non-adjacent legs of one side and the central leg of the opposite side alternate support of the robot. Thus, for a given position of the feet the central leg in support phase carries about half the robot's weight, while the two collateral legs in the support phase carry about one fourth of the robot's weight. A satisfactory force distribution and homogenization of the system was accomplished in the SILO6 design by displacing the central leg a little bit from the longitudinal body axis. In this case the central legs support lower weight and the corner legs increase their contribution in supporting the body. This results in the octagonal shape of the robot body (Figure 2).

The legs have been designed to be lightweight mechanisms which support the robot's weight and payload. Therefore, the

Table I Main walking robot features

\begin{tabular}{lcccc}
\hline \multirow{2}{*}{ Length } & \multicolumn{4}{c}{ Body dimensions (m) } \\
Width front/rear & Width middle & Height & Mass $(\mathrm{kg})$ \\
\hline $\mathbf{0 . 8 8}$ & 0.2 & 0.45 & 0.26 & 44.34 \\
\hline
\end{tabular}

Figure 2 SILO6 body dimensions and solar panel distribution

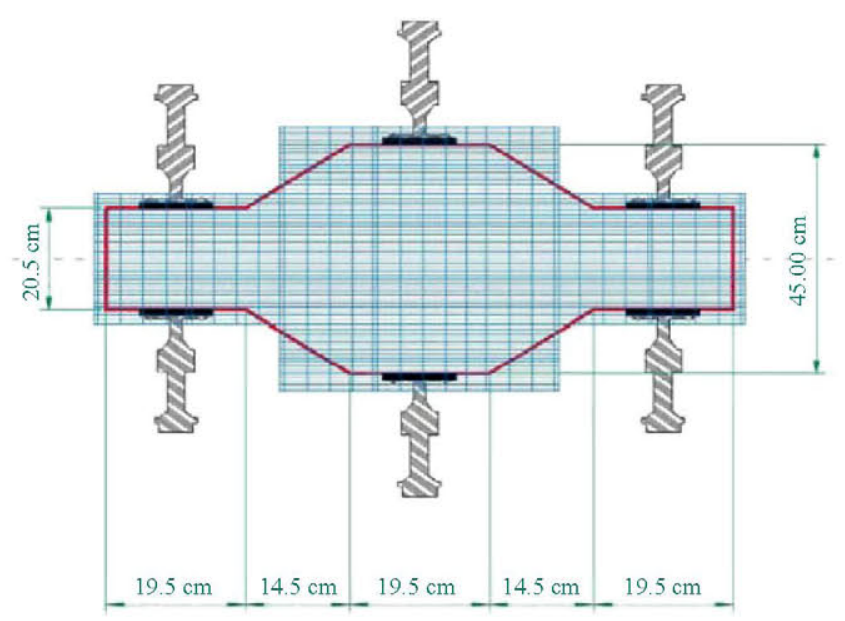


load carried by each leg is large and must be supported by the leg in different configurations. Each leg is actuated by three DC motors to drive the three joints of the leg.

\subsection{The scanning manipulator}

As mentioned above, the DYLEMA project requires a scanning device to sweep the sensor head across large areas. The easiest system would be a manipulator tailor-made for this task. Such a manipulator would require three DOFs to position the sensor in a $3 \mathrm{D}$ area; assuming that the system is scanning a non-flat area, motion in the $\mathrm{x}, \mathrm{y}$ and $\mathrm{z}$ components would be required. Additionally, the detector would have to be adapted to small terrain inclinations; hence, two additional DOFs would be needed at the wrist to control detector attitude. The metal detector has radial symmetry, so no additional DOFs would be needed for orientation control. To sum up, a manipulator with at least five DOFs is needed to accomplish the task. A manipulator designed with an $R R R$ arm configuration is sufficient for this application. Figure 3 shows a photograph of the scanning manipulator, which meets the design requirements. Joint 5 provides a $\pm 45^{\circ}$ pitch angle to the sensor head. Joint 4 gives a $\pm 200^{\circ}$ roll angle to let the system face the ground with either the sensor head or a grass cutter used to remove brushes in front of the sensor head. Joint 1 is an ordinary rotary joint and joints 2 and 3 , required to support the highest torques, are combined in a differential configuration around a typical three conical-pinion system. All joints are driven by DC motors. See Ponticelli et al. (2008) for a detailed mechanical description.

\subsection{Power supply}

The motion of the SILO6 robot is driven by a total of $18 \mathrm{DC}$ motors (three per leg). Added to the five DC motors that provide motion to the scanning manipulator means that the whole system is driven by 23 DC motors. There are also onboard electronics (control cards, power amplifiers, sensors, etc.) that require some power. The whole system is fed by three packs of Ion-Lithium rechargeable batteries that provide about 2 hours of autonomous operation. Seven elements of Amopack 7S1P MP 176065 batteries are packed to provide $24 \mathrm{VDC}(25.2 \mathrm{~V}$ nominal; $29.4 \mathrm{~V}$ maximum) with $5.8 \mathrm{~A} / \mathrm{h}$ capacity. The batteries are rechargeable at $29.4 \mathrm{~V}(4.1 \mathrm{~V} \pm$ $0.04 \mathrm{~V}$ per element) and $1.3 \mathrm{~A}$. Three packs of seven elements supply the DYLEMA system: one pack supplies power to the on-board computer and control cards and two packs are connected in parallel to supply all of the DC motors and

Figure 3 Scanning manipulator

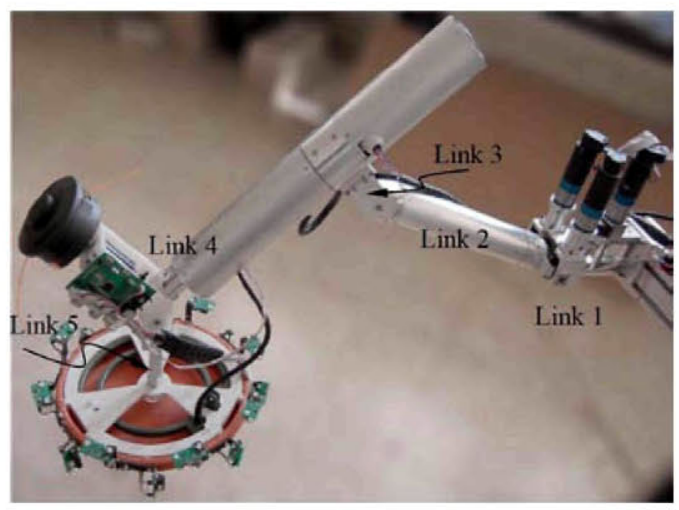

power electronics. The location of the battery packs on-board the SILO6 robot is shown in Figure 4.

\subsection{Energy autonomy in the DYLEMA project}

The current aim of phase three of the DYLEMA project is to provide energy autonomy to the complete system. Therefore, all the power will be supplied by solar cells. For this purpose, highly efficient solar cells have been selected. The AzureSpace solar cell with the $3 \mathrm{C} 35 / 175-100$ cell type based on GaInP/ $\mathrm{GaAs} / \mathrm{Ge}$ has an efficiency of 35.8 percent and provides a power of $17.92 \mathrm{~W}$. Also, a system of solar trackers is required to ensure that the focus of the sunlight falls directly on cells at any time of the day and that optimum performance of the cell is achieved at every moment. Maximum power consumption of the SILO6 robot and the manipulator has been measured and instantaneously reaches $300 \mathrm{~W}$. The solar panel's tracker system consumes $50 \mathrm{~W}$. Therefore, total power consumption is $350 \mathrm{~W}$ and 20 units of $3 \mathrm{C} 35 / 175-100$ cells are needed. Providing that amount of power solar cell encapsulation over the top of the SILO6 robot is shown in Figure 2. Solar panel cells and tracker system add an additional weight of $13 \mathrm{~kg}$. The legs have to support the whole weight of the robot, the manipulator and the solar panel, so the most efficient leg configuration has to be chosen in order to support this increased load and improve power consumption.

\section{Leg configurations}

In order to determine the most efficient configuration for a hexapod, two configurations have been implemented in the SILO6 robot, the insect and the mammal one. In the insectlike configuration, the axis of rotation of the first joint lies perpendicular to the longitudinal body axis and parallel to the axis $z_{b}$ of the body reference system, and the axes of rotation of the second and third joints lie perpendicular to the first one and parallel to the longitudinal body axis. In contrast, in the mammal-like configuration, the axis of rotation of the first joint lies perpendicular to the axis $z_{b}$ of the body reference system and parallel to the longitudinal body axis and the axes of rotation of the second and third joints lie perpendicular to the first one and to axis $x_{b}$ of the body reference system (Figure 5). These leg configurations are used for simulation and theoretical analysis and are implemented in the experimental platform SILO6 to compute energy consumption.

Figure 4 lon-Li batteries on-board the SILO6 robot

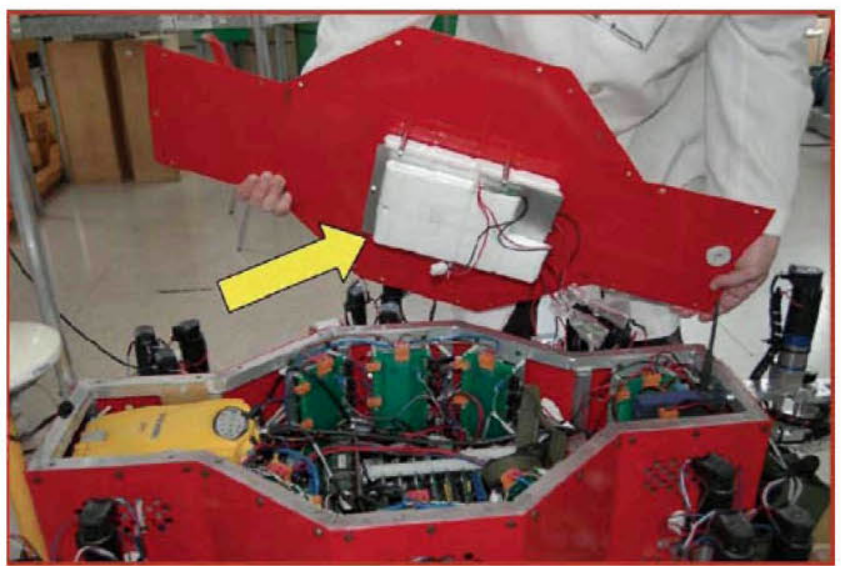


Figure 5 Leg configurations, joint-axis distribution and dimensions

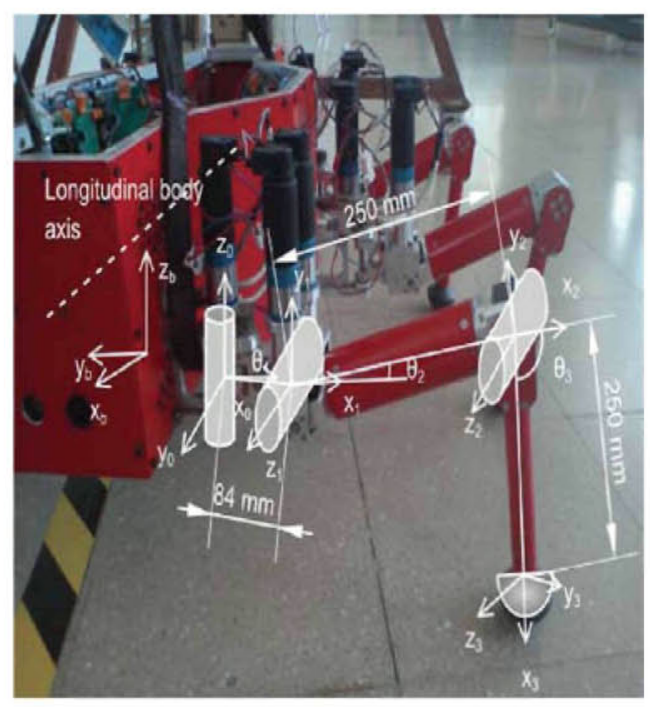

(a)

Notes: (a) Insect; (b) mammal

First, a theoretical analysis is performed making use of a model of power consumption and a dynamic simulation package in order to determine the sources of energy expenditure. Following that, the two configurations are implemented in the real robot, power consumption is acquired during real experiments and results are analyzed.

\section{Theoretical approach}

In this theoretical approach, the two configurations are analyzed in order to compare their energy efficiency. A nondimensional parameter is considered for comparison, evaluation and optimization purposes, which is the specific resistance. The specific resistance is a feature used in any kind of vehicle and is defined as (Siciliano and Khatib, 2008):

$$
\varepsilon=\frac{E}{W L}=\frac{P}{W v}
$$

where $E$ is the energy required to travel a distance $L$, by a vehicle with weight $W . P$ is the power consumed and $v$ is the speed of the vehicle. The specific resistance considers the power consumed per unit of mass and unit of speed. Therefore, it allows vehicle performance to be compared independently of weight and speed. For example, in a hexapod robot, the mammal configuration is slower than the insect one.

A walking robot can perform a variety of gaits featuring very different properties. It is well known that a hexapod performing an alternating-tripod gait can achieve its highest speed (Song and Waldron, 1989) and, due to the fact that statically stable walking robots are intrinsically slow, alternating tripods becomes the most usual gait for both natural and artificial hexapods. Thus, we focus our study on an alternating-tripod gait.

In order to compare the energy consumption of the two configurations a set of rules that optimize the gait will be established. This set of rules to optimize the energy consumption are based on three critical parameters of the gait (Figure 6):

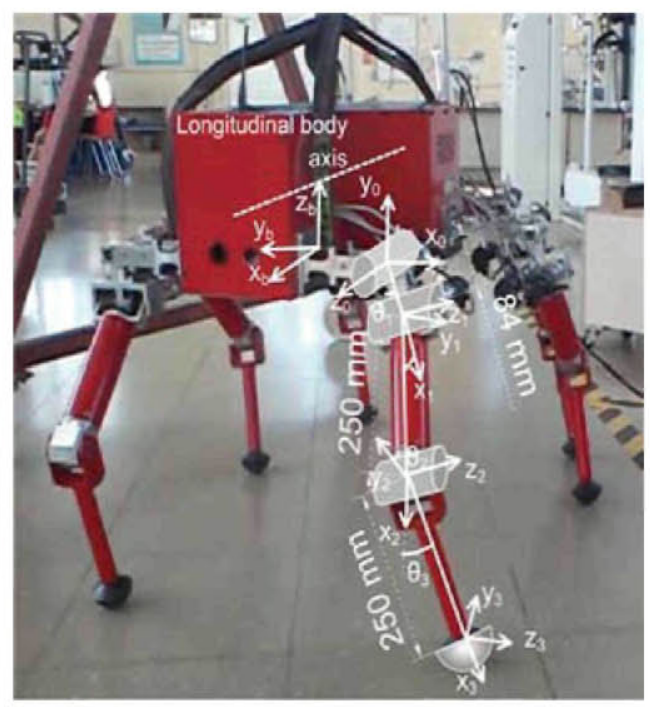

(b)

Figure 6 Gait parameters in a six-legged robot

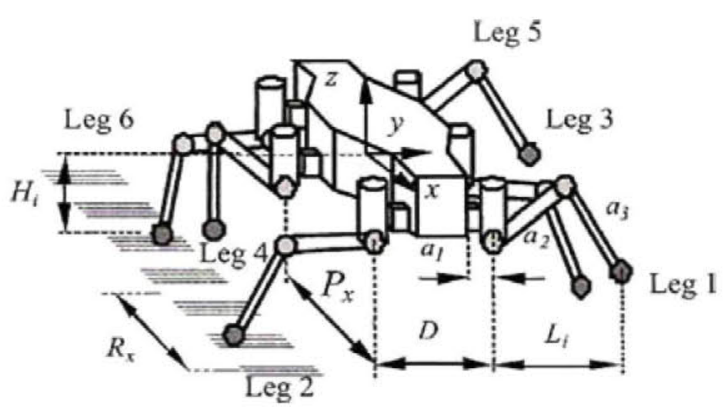

1 The distance of the footholds to the origin of the leg reference frame $L_{i}$.

2 The leg stroke $R_{x}$.

3 The body height of the robot, $H_{i}$.

These three parameters can change joint speed and torque, and therefore, power consumption. As a first approach, to establish the optimum values of the mentioned parameters for a given configuration, the mechanical power consumed is given by:

$$
P=\frac{\int_{0}^{T} \sum_{i=1}^{6} \sum_{i=1}^{3}\left(\omega_{i j}(t) \tau_{i j}(t)\right) d t}{T}
$$

where $\omega_{i j}(t)$ is the angular speed and $\tau_{i j}(t)$ is the torque at joint $j$ of leg $i$. Using the dynamics simulation package Yobotics! SCS (Yobotics Inc., 2002), joint speed and torque for each leg are obtained and inserted in equation (2) to compute the power consumed.

4.1 Parameter optimization in the insect configuration Marhefka and Orin (1997) establish some rules to optimize the gait in an insect configuration. Here these rules have been adapted to the critical parameters, as follows:

- Insect rule 1 (footholds and stroke length). The footholds should be set at points on an arc of a circle about the 
vertical hip axis, whose radius is determined so that the leg distal link is nearly vertical (Figure 7).

- Insect rule 2 (body height). To generate the required support forces with a minimum of joint torques (at joint 2), the body should be lowered so as to raise the knee above the hip.

\subsection{Parameter optimization in the mammal configuration}

The mammal configuration is not frequently implemented in hexapod robots, although some theoretical results can be found (Silva and Machado, 2008). By analyzing the power consumption on the dynamics simulation the following set of rules are established heuristically here:

- Mammal rule 1 (footholds). The footholds that minimize power consumption are those on the horizontal projection of the first joint on the ground. Thus, the lateral length should be $L_{i}=0$.

- Mammal rule 2 (stroke length). The foot-ground reaction force should pass through the hip to minimize joint torque. Also, stroke length must increase as the speed does.

- Mammal rule 3 (body height). Power consumption is reduced if the foot-ground reaction force passes through the knee, which is achieved if the robot walks as high as possible.

Following the rules above, optimum gait parameters have been applied to simulation and to real experiments. Table II shows the optimum critical values gathered after applying said rules and the specific resistance computed in a first approach using equations (1) and (2). As can be seen in this table, the insect configuration seems to be 25 percent more efficient than the mammal one when walking along a straight line. However, notice that power consumed by the motors has not been considered in this initial approach. The electro-mechanical model of power consumption will be addressed in the next section.

\section{Electro-mechanical analysis of power consumption}

The energy consumption in a robot is given by the sum of the energy consumed at every joint plus the energy consumed by electronic equipment (computers, drivers, analog I/O, etc.). The contribution of this last term is beyond of the scope of this work.

If a joint is driven by an electric motor and taking into account the well-known electrical model of a motor (Gonzalez de Santos et al., 2009), the energy consumed is given by:

$$
E(t)=\int u_{a p p}(t) i(t) d t=\int\left(\frac{k_{E}}{k_{M}} \omega(t) \tau(t)+\frac{R}{k_{M}^{2}} \tau^{2}(t)\right) d t
$$

where $u_{a p p}$ is the applied voltage, $i(t)$ is the current through the motor, $k_{E}$ is the back electromotive constant, $k_{M}$ is the torque constant, $R$ is the electric resistance of the motor, $\omega(t)$ is the motor speed and $\tau(t)$ is the motor torque. The relation between $k_{E}$ and $k_{M}$ for the motors in SILO6 is as follows:

$$
k_{E}=k_{M}\left(\frac{2 \pi}{60}\right)
$$

In equation (3) the term $k_{E} / k_{M}(\omega(t) \tau(t))$ is related to the mechanical energy and the term $R / k_{M}^{2}\left(\tau^{2}(t)\right)$ corresponds to electrical losses in the motor.

Taking the contribution of all joints in the hexapod into consideration and under the assumption that the negative mechanical energy is lost, because an electrical motor cannot store energy, the total energy becomes:

$$
E=\int_{0}^{T} \sum_{i=1}^{6} \sum_{i=1}^{3}\left(\Delta\left(\frac{k_{E}}{k_{M}} \omega_{m_{i j}}(t) \tau_{m_{i j}}(t)+\frac{R_{j}}{k_{M_{j}}^{2}} \tau_{m_{i j}}^{2}(t)\right)\right) d t
$$

where the function $\Delta(x)$ is defined as:

$$
\Delta(x)=\left\{\begin{array}{lll}
x & \text { if } & x>0 \\
0 & \text { if } & x \leq 0
\end{array}\right.
$$

Figure 7 (a) Leg distal link is nearly vertical and (b) points on an arc of a circle about the vertical hip axis

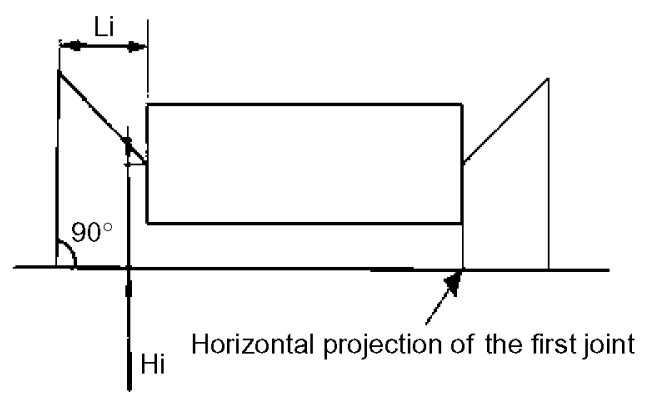

(a)

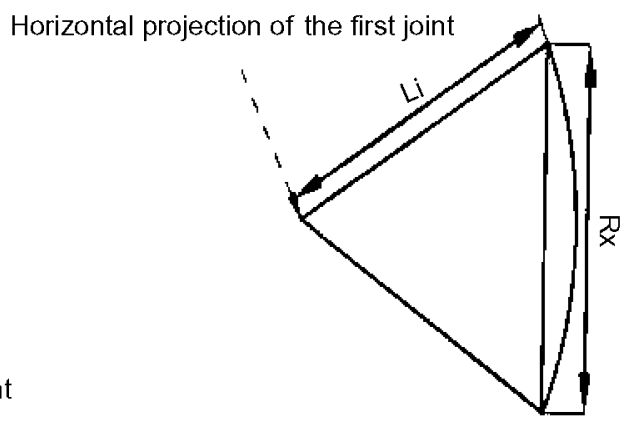

(b)

Table II Specific resistance of insect and mammal configurations with optimized gait parameters

\begin{tabular}{lcccc}
\hline Configuration & Lateral length $L_{i}(\mathrm{~m})$ & Stroke length $R_{x}(\mathrm{~m})$ & Body height $H_{i}(\mathrm{~m})$ & Specific resistance $\varepsilon$ \\
\hline Insect & 0.2 & 0.25 & 0.2 & 1.04 \\
Mammal & 0 & 0.3 & 0.52 & 1.37
\end{tabular}


Joint speeds and torques are directly obtained from the dynamic simulation, but equation (5) refers to the speeds and torques of the motors. Joint speed and torque and motor speed and torque are related through the mechanical and electrical parameters of the motor-gear system (motor-gear parameters for SILO6 are shown in Table III). The equations that relate both joint speed and torque with motor speed and torque are:

$$
\omega_{m}=N \omega_{s} \quad \tau_{m}=\mathscr{f} N \alpha_{s}+B N \omega_{s}+\frac{\tau_{s}}{\eta N}
$$

where $\mathcal{F}$ is the rotor inertia, $N$ is the gear ratio, $B$ is the friction constant, $\alpha_{s}$ in the joint's angular acceleration, $\omega_{s}$ and $\tau_{s}$ are joint angular speed and torque, respectively, and $\eta$ is the mechanical efficiency. Using the values obtained of $\alpha_{s}, \omega_{s}$ and $\tau_{s}$ in dynamic simulation, the energy consumption is computed. Also, from the terms in the right hand side of equation (3), it is possible to distinguish between mechanical consumption and electrical losses.

Table IV shows the results of applying the values obtained in dynamic simulation of the joint's angular acceleration, angular speed and torque to equations (5) and (7) for $0^{\circ}$ and for the $20^{\circ} \mathrm{crab}$ gait.

Through the observation of these tables some interesting conclusions are noted:

- Electrical consumption dominates over mechanical power; it takes 81 percent of the total power in the mammal configuration and 92 percent in the insect one.

- Regardless of whether mechanical power expenditure is still higher in the mammal configuration, electrical consumption is larger in the insect one. And, as electric consumption is more relevant than mechanical, the largest total power consumption occurs in the insect configuration.

- When the crab gait changes from a $0^{\circ}$ to a $20^{\circ} \mathrm{crab}$ angle, the mechanical power increases 34.2 percent in the insect configuration while in the mammal one there is not an important increase.

\section{Experimental analysis}

With the above theoretical analysis of energy consumption in hand, both configurations have been implemented in the robotic platform SILO6. The mechanical structure of the leg has been changed (Figure $5 \mathrm{a}$ and $\mathrm{b}$ ) to fit with the two configurations and the optimum gait parameters have been applied to compare power expenditure. The SILO6 robot is driven by $18 \mathrm{DC}$ motors (three per leg). There are also on-board electronics (control cards, power amplifiers, sensors, etc.) that require some power. In these experiments only the actuator's power consumption is measured, while the consumption of the on-board computer and sensors are assumed to be constant. Output current and voltage from the actuator's power supply is captured making use of a custom-made power measurement unit (PMU). These two analog measurements have been acquired through a PCMCIA National Instruments DAQCardA $1-16 \mathrm{XE}-50$ in a portable PC. The acquisition sampling time is $1 \mathrm{~ms}$. Figure 8 shows the experimental setup.

In order to estimate each term of the power consumption, three measurements have been carried out:

1 Power consumption of SILO6 stopped and suspended in the air (without touching the ground).

2 Power consumption of SILO6 walking in the air along a straight line in both configurations, the insect and the mammal.

3 Power consumption of SILO6 walking on flat, rigid ground along a straight line and in a $20^{\circ} \mathrm{crab}$ gait in both configurations, the insect and the mammal.

Experiment 1 provides the power losses in the power electronic cards. Experiment 2 provides power consumption for the leg movement. Finally, experiment 3 gives the power consumed to support the body. Table $\mathrm{V}$ shows the different sources of power consumption obtained in the experiment.

Analyzing this result considering the dynamic model of a leg structure, it is observed that the power consumed at leg motion is related with the leg dynamics during its transfer phase, following the expression $D(q) \ddot{q}+H(q, \dot{q})+C(q)$. Where $D(q)$ is the $3 \times 3$ mass matrix of the leg, $q$ is the vector of generalized coordinates, $H$ is a $3 \times 1$ vector of centrifugal and Coriolis terms, and $C(q)$ is a $3 \times 1$ vector of gravity terms. The measured power consumed to support the robot weight is related with the term $\mathcal{F}^{T} F$, where $F$ is the vector of the ground-contact forces and $\mathcal{F}$ is the Jacobian matrix.

Analyzing and comparing the experimental results it can be deduced that the insect configuration has less capacity to

Table III SILO6 joint parameters

\begin{tabular}{|c|c|c|c|c|c|}
\hline \multirow[b]{2}{*}{ Leg joint } & \multicolumn{2}{|c|}{ Motor } & \multicolumn{2}{|c|}{ Gear } & \multirow[b]{2}{*}{ Efficiency $\eta(\%)$} \\
\hline & Maximum torque $(\mathrm{mNm})$ & Torque constant $\mathrm{k}_{\mathrm{M}}(\mathrm{Nm} / \mathrm{A})$ & Resistance $R(\Omega)$ & Ratio N & \\
\hline 1 & 110 & 0.0333 & 0.62 & 246 & 63 \\
\hline 2 & 110 & 0.0333 & 0.62 & 881.5 & 42 \\
\hline 3 & 110 & 0.0333 & 0.62 & 881.5 & 42 \\
\hline
\end{tabular}

Table IV Mechanical, electrical and total consumed power in the configuration of insect and mammal walking along a straight line $\left(0^{\circ} \mathrm{crab}\right.$ gait) and in a $20^{\circ} \mathrm{crab}$ gait

\begin{tabular}{lccccc}
\hline Configuration & Crab angle & Mechanical power $(\mathrm{W})$ & Electrical power $(\mathrm{W})$ & Total power $(\mathrm{W})$ & Specific resistance $\varepsilon$ \\
\hline Insect & $0^{\circ}$ & 2.28 & 37.96 & 40.25 & 5.31 \\
Mammal & $0^{\circ}$ & 5.50 & 23.81 & 29.32 & 3.65 \\
Insect & $20^{\circ}$ & 3.06 & 38.51 & 41.58 & 5.48 \\
Mammal & $20^{\circ}$ & 5.50 & 24.5 & 30.01 & 3.73
\end{tabular}


Figure 8 Experimental setup to measure SILO6 power consumption

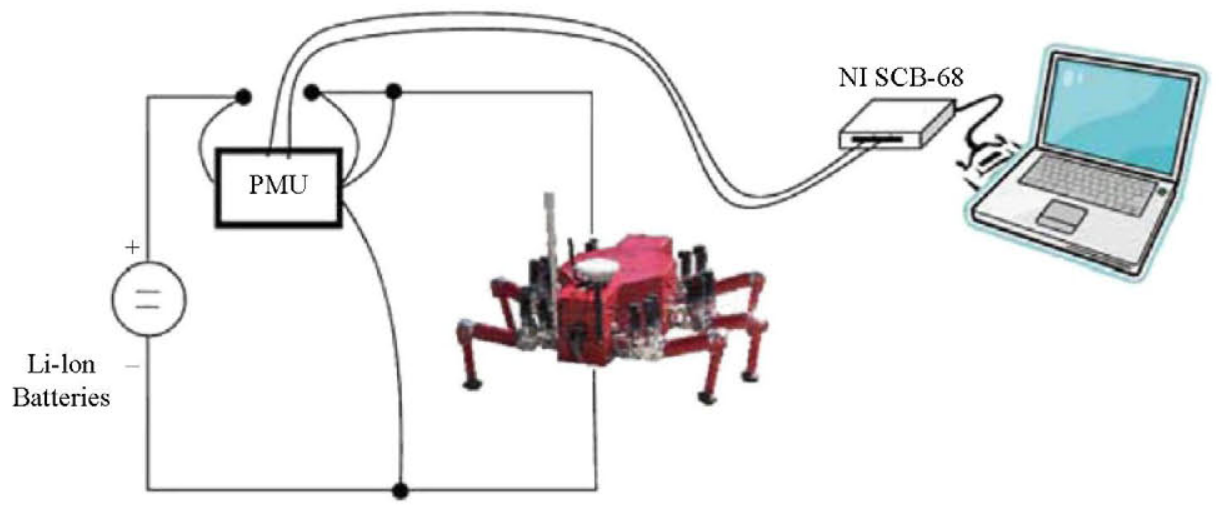

Table V Mechanical, electrical and total consumed power in the configuration of insect and mammal walking in a $0^{\circ}$ and $20^{\circ} \mathrm{crab}$ gait

\begin{tabular}{|c|c|c|c|c|c|c|}
\hline \multirow[b]{2}{*}{ Configuration } & \multirow[b]{2}{*}{ Gait } & \multicolumn{5}{|c|}{ Power consumption } \\
\hline & & Power electronic cards (W) & Leg motion $(W)$ & Body support (W) & Actuators (W) & Total $(\mathrm{W})$ \\
\hline \multirow[t]{2}{*}{ Insect } & Crab $0^{\circ}$ & 40.89 & 17.36 & 6.9 & 24.26 & 65.15 \\
\hline & Crab $20^{\circ}$ & 40.89 & 18.89 & 12.29 & 31.18 & 72.07 \\
\hline \multirow[t]{2}{*}{ Mammal } & Crab $0^{\circ}$ & 40.89 & 20.02 & 3.67 & 23.89 & 64.58 \\
\hline & Crab $20^{\circ}$ & 40.89 & 22.23 & 5.55 & 27.79 & 68.67 \\
\hline
\end{tabular}

support static loads, but conversely the power consumption due to leg dynamics is lower in the insect configuration than in the mammal one. Also notice that, in crab gaits, the mammal configuration features lower power consumption.

Some clear differences between insect and mammalian walking in the theoretical calculations became very much smaller in the practical evaluations. Those quantitative differences are mainly due to friction and backlashes that in theoretical calculations have not been taken into account because of the complexity of simulating them. Moreover, in the simulation, parameter alpha is not computed and it had to be estimated in the equations that relate joint speed and torque with motor speed and torque.

\section{Results and conclusions}

It is widely accepted that the mammal configuration in statically stable walking machines is better for supporting high loads while the insect one is considered to improve mobility (Todd, 1985). This paper presents a theoretical and experimental analysis of power consumed by an hexapod robot in both configurations. To allow for a thorough analysis, a non-dimensional parameter has been used for comparison of power consumption. Also, rules for optimizing the two configurations have been proposed in order to compare the insect and the mammal configuration in their most efficient disposition. After the results shown in this paper, it can be stated that this statement is valid only if static loads are taken into account, that is, the power consumption for supporting the body and its pay-load is larger in an insect configuration. However, as is shown in this paper the leg dynamics is not negligible even in statically stable locomotion and its contribution to power consumption is even greater than the static load one. Also, it is important to note that the larger contribution to the power consumed in a walking robot is due to the electric term (larger than 80 percent). Some results are clearly generalizable, e.g. it will be energy efficient that the ground reaction force pass through some joint. Finally, taking the effect of the gait crab angle into account, the mammal configuration features lower consumption in a crab gait. In a natural environment, such as the one found in demining applications, a robot has to change its trajectory constantly; thus based on the point of view of the energy consumption, the better configuration for this applications is the mammal one. The robot energy consumption could be also improved if the motor's efficiency improves likewise.

\section{References}

Baudoin, Y. (2005), "Mobile robotics systems facing the humanitarian demining problem: state of the art, technical report task", International Test and Evaluation Program for Humanitarian Demining, The Royal Military Academy of Belgium (RMA), Brussels.

Baudoin, Y., Acheroy, M., Piette, M. and Salmon, J.P. (1999), "Humanitarian demining and robotics", Mine Action Information Center fournal, Vol. 3 No. 2.

Gonzalez de Santos, P., Garcia, E. and Estremera, J. (2006), Quadrupedal Locomotion: An Introduction to the Control of Four-Legged Robots, Springer, London.

Gonzalez de Santos, P., Galvez, J.A., Estremera, J. and Garcia, E. (2003), "SILO4 - a true walking robot for the comparative study of walking machine techniques", IEEE Robotics and Automation Magazine, Vol. 10 No. 4, pp. 23-32.

Gonzalez de Santos, P., Garcia, E., Ponticelli, R. and Armada, M. (2009), "Minimizing energy consumption in hexapod robots", Advanced Robotics, Vol. 23, pp. 681-704. 
Gray, J. and Murray, D. (2003), "The aftermath of war: landmines", Edge Research Paper.

Hirose, S. and Kato, K. (1998), "Quadruped walking robot to perform mine detection and removal task", Proceedings of the 1st International Conference on Climbing and Walking Robots, Brussels, Belgium, pp. 261-6.

Hirose, S., Yokota, S., Torii, A., Ogata, M., Sugamuna, S. and Takita, K. (2005), Quadruped Walking Robot Centered Demining System - Development of TITAN IX and its Operation, ICRA, New Delhi, pp. 1296-302.

Jimenez, M.A. and Gonzalez de Santos, P. (1996), "Attitude and position control method for realistic legged vehicles", Robotics and Autonomous Systems, Vol. 18 No. 3, pp. 345-54.

Marhefka, D.W. and Orin, D.E. (1997), "Gait planning for energy efficiency in walking machines", IEEE Robotics and Automation Magazine, Vol. 97, April, pp. 474-8.

Nonami, K., Huang, Q.J., Komizo, D., Shimoi, N. and Uchida, H. (2000), "Humanitarian mine detection sixlegged walking robot", Third International Conference on Climbing and Walking Robots (CLAWAR'OO), Madrid, Spain, pp. 861-8.

Oku, M., Koseki, H., Ohroku, H., Harada, Y., Futagami, K., Tran, D.C., Li, L., Lin, X., Sakai, S. and Nonami, K. (2008), "Rough terrain locomotion control of hydraulically actuated hexapod robot COMET-IV", Proceedings of 2008 FSME Conference on Robotics and Mechatronics (ROBOMEC 2008), Nagano, fapan (in Japanese).

Ponticelli, R., Garcia, E., Gonzalez de Santos, P. and Armada, M. (2008), "A scanning robotic system for humanitarian de-mining activities", Industrial Robot, Vol. 35 No. 2, pp. 133-42.

Rizo, J., Coronado, J., Campo, C., Forero, A., Otalora, C. and Devy, M. (2003), Ursula: Robotic Demining System, ICAR, New Delhi, pp. 538-43.

Siciliano, B. and Khatib, O. (2008), Springer Handbook of Robotics, Springer, London.

Silva, M.F. and Machado, J.A.T. (2008), "Kinematic and dynamic performance analysis of artificial legged systems", Robotica, Vol. 26 No. 1, pp. 19-40.

Song, S.M. and Waldron, K.J. (1989), Machines That Walk: The Adaptive Suspension Vehicle, The MIT Press, Cambridge, MA.

Todd, D.J. (1985), Walking Machines: An Introduction to Legged Robots, Kogan Page Ltd, London.

Voth, D. (2002), "Nature's guide to robot design", IEEE Intelligent Systems, pp. 4-7.

Waldron, K.J., Vohnout, V.J., Pery, A. and McGhee, R.B. (1984), "The adaptive suspension vehicle", The International fournal of Robotics Research, Vol. 3 No. 2, pp. 37-42.

Yobotics Inc. (2002), Yobotics! Simulation Construction Set: Users Guide, Yobotics Inc., Boston, MA, available at: www. yobotics.com/

\section{Corresponding author}

D. Sanz-Merodio can be contacted at: daniel.sanz@car. upm-csic.es 